\title{
Stochastic $H_{\infty}$ Control for Discrete-Time Singular Systems with State and Disturbance Dependent Noise
}

\author{
Yong Zhao, ${ }^{1,2}$ Xiushan Jiang, ${ }^{3}$ and Weihai Zhang ${ }^{1}$ \\ ${ }^{1}$ College of Electrical Engineering and Automation, Shandong University of Science and Technology, Qingdao 266590, China \\ ${ }^{2}$ College of Mathematics and Systems Science, Shandong University of Science and Technology, Qingdao 266590, China \\ ${ }^{3}$ College of Information and Control Engineering, China University of Petroleum (East China), Qingdao 266580, China
}

Correspondence should be addressed to Weihai Zhang; w_hzhang@163.com

Received 24 August 2016; Revised 9 January 2017; Accepted 1 March 2017; Published 29 March 2017

Academic Editor: Ciprian G. Gal

Copyright (C) 2017 Yong Zhao et al. This is an open access article distributed under the Creative Commons Attribution License, which permits unrestricted use, distribution, and reproduction in any medium, provided the original work is properly cited.

\begin{abstract}
This paper is concerned with the stochastic $H_{\infty}$ state feedback control problem for a class of discrete-time singular systems with state and disturbance dependent noise. Two stochastic bounded real lemmas (SBRLs) are proposed via strict linear matrix inequalities (LMIs). Based on the obtained SBRLs, a state feedback $H_{\infty}$ controller is presented, which not only guarantees the resulting closedloop system to be mean square admissible but also satisfies a prescribed $H_{\infty}$ performance level. A numerical example is finally given to illustrate the effectiveness of the proposed theoretical results.
\end{abstract}

\section{Introduction}

Because of extensive applications in economy, mechanical systems, systems with human operators, and so on, a great deal of attention has been paid to a class of stochastic linear systems governed by Itô's stochastic differential equations over the past years [1-4]. In particular, there is an increasing interest in the $H_{\infty}$ control and mixed $H_{2} / H_{\infty}$ control [5-10].

On the other hand, singular systems, as it is well known, are ideal mathematical models in practical modeling such as in electric circuits, economics, and aircraft modeling [11-13]. It is noted that the study on the control of deterministic or Markov jump singular systems has made fruitful achievement on stability $[14,15]$, stabilization $[16,17], H_{\infty}$ control $[18-$ 25], and $H_{2} / H_{\infty}$ control [26], as well as sliding mode control $[27,28]$. However, it can be seen that, owing to the presence of the diffusion term, few works have been done on stochastic singular systems with state-dependent noise so far. Different from deterministic singular systems or Markov jump singular systems without multiplicative noise, regularity, as a necessary and sufficient condition for the existence of the solution to singular systems, is not applicable to stochastic singular systems. For this reason, some researchers regarded the existence of impulse-free solutions as a precondition [29, 30]; others provided suitable conditions to guarantee the existence of an impulse-free solution [31-34]. Under the assumption that $\operatorname{deg}(\operatorname{det}(s E-$ $A))=\operatorname{rank}(E), \operatorname{det}(s E-A) \neq 0$ and $\operatorname{rank}\left(E, A_{0}\right)=\operatorname{rank}(E)$, the filtering problem was firstly investigated in [31, 32]. Then, [34] removed the assumption condition $\operatorname{deg}(\operatorname{det}(s E-$ $A))=\operatorname{rank}(E)$ and discussed the observer-based controller design by means of a sequential design technique. Based on the assumption proposed by [34], the problem of robust finite-time $H_{\infty}$ control for a class of singular stochastic systems with parametric uncertainties was further discussed in [35], and the state feedback controller ensuring that the closed-loop system was robust stochastically bounded with a prescribed $H_{\infty}$ performance level was designed by means of the linear matrix inequalities (LMIs). Recently, the assumption condition was reduced to $\operatorname{rank}\left(E, A_{0}\right)=\operatorname{rank}(E)$ in [36-38], and sufficient conditions for the mean square admissibility of stochastic singular systems were proposed in terms of strict LMIs. Furthermore, the state feedback $H_{\infty}$ control for continuous-time singular stochastic systems was investigated in [39]. To the best of our knowledge, all the results which appeared in [29-35, 38, 39] were given in continuous-time setting except for $[36,37]$. In particular, the $H_{\infty}$ state feedback control problem for discrete-time singular 
systems with state and disturbance dependent noise has never been tackled. In fact, there are some differences in studying the $H_{\infty}$ control problem between discrete-time stochastic singular systems and continuous-time stochastic singular systems. For instance, how to deal with the difficulties arising from the nonlinear terms when employing the state feedback controller to the system? How to provide conditions to guarantee the irrelevance of the state $x(k)$ and the sequence of the random variables $\{w(k)\}_{k \geq 0}$ ? So, it is very meaningful to discuss the issue of the $H_{\infty}$ control for discrete-time stochastic singular systems.

In this paper, motivated by [37], we investigate stochastic $H_{\infty}$ state feedback control problem for discrete-time singular systems with state and disturbance dependent noise. Compared to our earlier work $[36,37]$ and other available works in the literature, the main contributions of this paper lie in the following aspects. (i) By introducing the slack variables approach proposed in $[22,23]$, the new stochastic admissibility conditions for singular stochastic system are firstly provided, which are different from those in $[36,37]$ and are more helpful to derive the SBRLS. (ii) The suitable LMI conditions are presented, which not only meet the requirement of the mean square admissibility for the singular stochastic system but also ensure the irrelevance of the state $x(k)$ and the sequence of the random variables $\{w(k)\}_{k \geq 0}$, while the above irrelevance is crucial to discuss the control problem of discrete-time stochastic systems. (iii) Two LMIbased SBRLs are derived for discrete-time stochastic singular systems with state and disturbance dependent noise, which can be regarded as extensions of corresponding results on deterministic singular systems $[21,22]$ and normal stochastic systems $[5,8,9]$. Based on the obtained SBRLS, the state feedback $H_{\infty}$ controller is designed.

The remainder of this paper is organized as follows: Section 2 contains some fundamental notions and preliminary results. In Section 3, two SBRLS are obtained in terms of strict LMIs. In Section 4, the state feedback $H_{\infty}$ controller for singular stochastic systems is designed by using the augmented system. Section 5 gives a simulation example to illustrate the effectiveness of the proposed theoretical results. Conclusions are presented in Section 6.

Notations. $S_{n}$ is the set of all $n \times n$ symmetric matrices; $A>0$ (resp., $A<0$ ): $A$ is a real symmetric positive definite (resp., negative definite) matrix; $A^{T}$ is the transpose of $A ;\|\cdot\|$ is the Euclidean norm of a vector; $\mathscr{E}(\cdot)$ is the expectation operator; $I_{n}$ is the $n \times n$ identity matrix; $\mathrm{He}(A):=A+A^{T} ; N:=\{0,1, \ldots\}$. For a matrix $X \in R^{n \times m}$ with $\operatorname{rank}(X)=r$, let $X^{\perp} \in R^{(n-r) \times n}$ be any matrix with full row rank satisfying $X^{\perp} X=0$ and $X^{\perp}\left(X^{\perp}\right)^{T}>0$.

\section{Preliminaries}

Consider the following discrete-time stochastic singular system:

$$
\begin{aligned}
E x(k+1)= & A x(k)+A_{0} x(k) w(k)+B_{1} v(k) \\
& +B_{0} v(k) w(k)+B u(k),
\end{aligned}
$$

$$
\begin{aligned}
z(k) & =C x(k)+D v(k), \\
E x(0) & =x_{0} \in R^{n},
\end{aligned}
$$$$
k \in N \text {, }
$$

where $x(k)$ (or $x\left(k, x_{0}, v\right)$ for clear) $\in R^{n}, v(k) \in R^{q}, u(k) \in$ $R^{m}$, and $z(k) \in R^{p}$ are, respectively, the system state, the disturbance signal, the controlled input, and the controlled output. $A, A_{0}, B_{0}, B_{1}, B, C$, and $D$ are constant matrices of appropriate dimensions. The matrix $E$ may be singular and we assume that $\operatorname{rank}(E)=r \leq n . E x(0)=x_{0} \in R^{n}$ is the initial condition which is deterministic. Suppose $v(k)$ and $w(k)$ are uncorrelated, and $\{w(k)\}_{k \geq 0}$ is a sequence of real independent random variables defined on a complete probability space $\{\Omega, F, \mu\}$ with $\mathscr{E}(w(k))=0$ and $\mathscr{E}(w(s) w(t))=\delta_{s t}$ a Kronecker function. We denote by $F_{k}$ the $\sigma$-algebra generated by $w(0), \ldots, w(k)$; that is, $F_{k}=\sigma(w(s): 0 \leq s \leq k)$. Let $L^{2}\left(\Omega, R^{k}\right)$ represent the space of $R^{k}$-valued random vectors $\xi$ with $\mathscr{E}\|\xi\|^{2}<\infty . l_{w}^{2}\left(N, R^{k}\right)$ denote the set of nonanticipative square summable stochastic processes $y=\{y(k): y(k) \epsilon$ $\left.R^{k}\right\}_{k \in N}$, such that $y(k) \in L^{2}\left(\Omega, R^{k}\right)$ is $F_{k-1}$ measurable, where we define $F_{-1}=\{\emptyset, \Omega\}$; that is, $y(0)$ is a constant. The $l^{2}$-norm of $y \in l_{w}^{2}\left(N, R^{k}\right)$ is defined by

$$
\|y(\cdot)\|_{l_{w}^{2}\left(N, R^{k}\right)}=\left(\sum_{k=0}^{\infty} \mathscr{E}\|y(k)\|^{2}\right)^{1 / 2} .
$$

Lemma 1 (see [31]). If there are a pair of nonsingular matrices $U \in R^{n \times n}$ and $V \in R^{n \times n}$ for the triplet $\left(E, A, A_{0}\right)$ such that the following condition is satisfied, then system (1) with $u(k)=$ $0, v(k)=0$ has a unique causal solution.

$$
\begin{gathered}
U E V=\left[\begin{array}{ll}
I_{r} & 0 \\
0 & 0
\end{array}\right], \\
U A V=\left[\begin{array}{cc}
A_{1} & 0 \\
0 & I_{n-r}
\end{array}\right], \\
U A_{0} V=\left[\begin{array}{cc}
A_{01} & A_{02} \\
0 & 0
\end{array}\right],
\end{gathered}
$$

where $A_{1}, A_{01} \in R^{r \times r}, A_{02} \in R^{r \times(n-r)}$.

The condition for the existence and uniqueness of the solution to stochastic singular system (1) is given in Lemma 1, which is a basic requirement in system analysis and control synthesis.

Definition 2. System (1) with $u(k)=0$ and $v(k)=0$ is said to be

(i) asymptotically stable in the mean square if, for any initial condition $x_{0} \in R^{n}, \lim _{k \rightarrow \infty} \mathscr{E}\left\{\|x(k)\|^{2}\right\}=0$;

(ii) mean square admissible if it has a unique and causal solution and asymptotically stable in the mean square. 
Definition 3. The perturbed operator of system (1) with $u(k)=0$ is defined by $L: l_{w}^{2}\left(N, R^{q}\right) \rightarrow l_{w}^{2}\left(N, R^{p}\right)$,

$$
\begin{aligned}
L v(k):=C x(k ; 0, v)+D v(k), & \\
& \forall v(k) \in l_{w}^{2}\left(N, R^{q}\right), x_{0}=0,
\end{aligned}
$$

with its norm

$$
\begin{aligned}
\|L\|=\sup _{v \in l_{w}^{2}\left(N, R^{q}\right), v \neq 0, x_{0}=0} \frac{\|z(k)\|_{l_{w}^{2}\left(N, R^{p}\right)}}{\|v(k)\|_{l_{w}^{2}\left(N, R^{q}\right)}} \\
=\sup _{v \in l_{w}^{2}\left(N, R^{q}\right), v \neq 0, x_{0}=0} \frac{\left(\sum_{k=0}^{\infty} \mathscr{E}\|C x(k)+D x(k)\|^{2}\right)^{1 / 2}}{\left(\sum_{k=0}^{\infty} \mathscr{E}\|v(k)\|^{2}\right)^{1 / 2}} .
\end{aligned}
$$

Definition 4. System (1) with $u(k)=0$ is said to be mean square admissible and $\|L\|<\gamma$ if it is mean square admissible for $v(k)=0$ and $\|L\|<\gamma$ for $v(k) \neq 0$.

Assumption 5. In system (1), there exist nonsingular matrices $U, V$ such that the triplet $\left(E, A_{0}, B_{0}\right)$ satisfies the following condition:

$$
\begin{gathered}
U E V=\left[\begin{array}{ll}
I_{r} & 0 \\
0 & 0
\end{array}\right], \\
U A_{0} V=\left[\begin{array}{cc}
\widehat{A}_{01} & \widehat{A}_{02} \\
0 & 0
\end{array}\right],
\end{gathered}
$$

$$
U B_{0}=\left[\begin{array}{c}
\widehat{B}_{01} \\
0
\end{array}\right]
$$

where $\widehat{A}_{01} \in R^{r \times r}, \widehat{A}_{02} \in R^{r \times(n-r)}, \widehat{B}_{01} \in R^{r \times q}$.

Lemma 6. If there exists a matrix $P=P^{T}$ such that the following LMIs hold,

$$
\begin{aligned}
E^{T} P E & \geq 0, \\
A^{T} P A+A_{0}^{T} P A_{0}-E^{T} P E & <0,
\end{aligned}
$$

then one can obtain the following:

(i) System (1) with $u(k)=0$ and $v(k)=0$ is mean square admissible.

(ii) For system (1) with $u(k)=0$, the state $x(k)$ is uncorrelated with the sequence of real independent random variables $\{w(k)\}_{k \geq 0}$.

Proof. See the Appendix.

Theorem 7. System (1) with $u(k)=0$ and $v(k)=0$ is mean square admissible if one of the following equivalent statements holds.

(i) There exist matrices $P>0, Q=Q^{T}$ such that the following LMI holds:

$$
\begin{aligned}
A^{T}( & \left.P-E^{\perp^{T}} Q E^{\perp}\right) A+A_{0}^{T}\left(P-E^{\perp^{T}} Q E^{\perp}\right) A_{0} \\
& -E^{T} P E<0 .
\end{aligned}
$$

(ii) There exist matrices $P>0, Q=Q^{T}, F$ and $G$ such that the following LMI holds:

$$
\left[\begin{array}{cc}
-E^{T} P E+F A+A^{T} F^{T}+A_{0}^{T}\left(P-E^{\perp^{T}} Q E^{\perp}\right) A_{0} & -F+A^{T} G^{T} \\
* & P-E^{\perp^{T}} Q E^{\perp}-G-G^{T}
\end{array}\right]<0 .
$$

Proof. It is easy to see that conditions (7) and (8) are satisfied by setting $X=P-E^{\perp^{T}} Q E^{\perp}$ in (9). Therefore, the mean square admissibility of system (1) with $u(k)=0$ and $v(k)=0$ is obviously obtained by Lemma 6-(i). Similar to the proof of Theorem 4 in [22], we can verify that condition (9) is equivalent to condition (10).

Remark 8. Item (i) of Lemma 6 contains an equality constraint condition that is not easy to be checked numerically. To solve this problem, strict LMI conditions in Theorem 7 are introduced. Moreover, it is worth mentioning that conditions (9) and (10) are completely different from those in Theorem 4 of [36], which not only generalize the conditions of Theorem 1 [21] and Theorem 4 [22] to the stochastic case but also pave the way for developing SBRL. While item (ii) of Lemma 6 is crucial to discuss the control problem of discrete-time stochastic systems, see, for example, $[5,8,9]$.

\section{Stochastic Bounded Real Lemmas}

In this section, we aim to develop two SBRLs for system (1) in terms of LMIs. It is well known that SBRL plays an important role in the study of stochastic $H_{\infty}$ control and estimation.

Lemma 9. In system (1) with $u(k)=0, \forall T \in N, P=P^{T}$, and $x_{0} \in R^{n}$, one has

$$
\sum_{k=0}^{T} \mathscr{E}\left\{\left[\begin{array}{l}
x(k) \\
v(k)
\end{array}\right]^{T} R(P)\left[\begin{array}{l}
x(k) \\
v(k)
\end{array}\right]\right\}
$$




$$
\begin{aligned}
= & \mathscr{E}\left[x^{T}(T+1) E^{T} P E x(T+1)\right] \\
& -x^{T}(0) E^{T} P E x(0),
\end{aligned}
$$

where

$$
\begin{aligned}
& R(P) \\
& \quad=\left[\begin{array}{cc}
A^{T} P A+A_{0}^{T} P A_{0}-E^{T} P E & A^{T} P B_{1}+A_{0}^{T} P B_{0} \\
B_{1}^{T} P A+B_{0}^{T} P A_{0} & B_{1}^{T} P B_{1}+B_{0}^{T} P B_{0}
\end{array}\right] .
\end{aligned}
$$

Proof. By Lemma 6-(ii), $A x(k)+B_{1} v(k)$ and $A_{0} x(k)+B_{0} v(k)$ are uncorrelated with $w(k)$, and $\mathscr{E}\{w(k)\}=0$. Thus we have

$$
\begin{aligned}
\mathscr{E} & \left\{\left[A x(k)+B_{1} v(k)\right]^{T} P\left[A_{0} x(k)+B_{0} v(k)\right] w(k)\right\} \\
& =\mathscr{E}\left\{\left[A_{0} x(k)+B_{0} v(k)\right]^{T} P\left[A x(k)+B_{1} v(k)\right]\right. \\
\cdot & w(k)\}=0 .
\end{aligned}
$$

From (1) and (13), it follows that

$$
\begin{aligned}
\mathscr{E} & {\left[x^{T}(k+1) E^{T} P E x(k+1)-x^{T}(k) E^{T} P E x(k)\right] } \\
= & \mathscr{E}\left\{\left[A x(k)+B_{1} v(k)\right]^{T} P\left[A x(k)+B_{1} v(k)\right]\right\} \\
& +\mathscr{E}\left\{\left[A_{0} x(k)+B_{0} v(k)\right]^{T} P\left[A_{0} x(k)+B_{0} v(k)\right]\right\}
\end{aligned}
$$

$$
\begin{aligned}
& -\mathscr{E}\left[x^{T}(k) E^{T} \operatorname{PEx}(k)\right] \\
= & \mathscr{E}\left\{\left[\begin{array}{l}
x(k) \\
v(k)
\end{array}\right]^{T} R(P)\left[\begin{array}{c}
x(k) \\
v(k)
\end{array}\right]\right\} .
\end{aligned}
$$

Note that the following equality holds:

$$
\begin{aligned}
\mathscr{E} & {\left[x^{T}(T+1) E^{T} P E x(T+1)\right]-x^{T}(0) E^{T} P E x(0) } \\
& =\sum_{t=0}^{T} \mathscr{E}\left[x^{T}(k+1) E^{T} P E x(k+1)\right. \\
& \left.-x^{T}(k) E^{T} P E x(k)\right] .
\end{aligned}
$$

It is shown that (14) and (15) result in (11).

Lemma 10. In system (1) with $u(k)=0$, suppose $T \in N$ is given and $P=P^{T}$. Then, for any $x_{0} \in R^{n}, v(k) \in l_{w}^{2}\left(N, R^{q}\right)$, one has

$$
\begin{aligned}
J^{T}\left(x_{0}, v\right):= & \sum_{k=0}^{T} \mathscr{E}\left[\|z(k)\|^{2}-\gamma^{2}\|v(k)\|^{2}\right] \\
= & x_{0}^{T} P x_{0}-\mathscr{E}\left[x^{T}(T+1) E^{T} P \operatorname{Ex}(T+1)\right] \\
& +\sum_{t=0}^{T} \mathscr{E}\left\{\left[\begin{array}{c}
x(k) \\
v(k)
\end{array}\right]^{T} L(P)\left[\begin{array}{c}
x(k) \\
v(k)
\end{array}\right]\right\},
\end{aligned}
$$

where

$$
L(P)=\left[\begin{array}{cc}
A^{T} P A+A_{0}^{T} P A_{0}-E^{T} P E+C^{T} C & A^{T} P B_{1}+A_{0}^{T} P B_{0}+C^{T} D \\
* & -\gamma^{2} I+B_{1}^{T} P B_{1}+B_{0}^{T} P B_{0}+D^{T} D
\end{array}\right] .
$$

Proof. From Lemma 9, it follows that

$$
+\sum_{t=0}^{T} \mathscr{E}\left\{\left[\begin{array}{l}
x(k) \\
v(k)
\end{array}\right]^{T} L(P)\left[\begin{array}{l}
x(k) \\
v(k)
\end{array}\right]\right\}
$$

$$
\begin{aligned}
J^{T} & \left(x_{0}, v\right):=\sum_{k=0}^{T} \mathscr{E}\left[\|z(k)\|^{2}-\gamma^{2}\|v(k)\|^{2}\right] \\
& =\sum_{k=0}^{T} \mathscr{E}\left[x^{T}(k) C^{T} C x(k)+x^{T}(k) C^{T} D v(k)\right. \\
& \left.+v^{T}(k) D^{T} C x(k)+v^{T}(k)\left(D^{T} D-\gamma^{2} I\right) v(k)\right] \\
& +x^{T}(0) E^{T} P E x(0)-\mathscr{E}\left[x^{T}(T+1) E^{T} P E x(T\right. \\
& +1)]+\sum_{t=0}^{T} \mathscr{E}\left\{\left[\begin{array}{l}
x(k) \\
v(k)
\end{array}\right]^{T} R(P)\left[\begin{array}{l}
x(k) \\
v(k)
\end{array}\right]\right\}=x^{T}(0) \\
& \cdot E^{T} P E x(0)-\mathscr{E}\left[x^{T}(T+1) E^{T} P E x(T+1)\right]
\end{aligned}
$$

Theorem 11 (SBRL). System (1) with $u(k)=0$ is mean square admissible and $\|L\|<\gamma$ for given $\gamma>0$ if there exists a matrix $P=P^{T}$ such that the following LMIs,

$$
\begin{aligned}
E^{T} P E & \geq 0, \\
L(P) & <0,
\end{aligned}
$$

hold, where $L(P)$ is defined in Lemma 10.

Proof. If there exists a matrix $P=P^{T}$ such that $L(P)<0$, it is easy to see that $A^{T} P A+A_{0}^{T} P A_{0}-E^{T} P E<0$. This condition 
together with $E^{T} P E \geq 0$ in (19) can deduce that system (1) is mean square admissible. Below, we set about to prove $\|L\|<\gamma$ for given $\gamma>0$. To this end, we choose $\varepsilon>0$ sufficiently small such that $L(P) \leq-\varepsilon^{2} I_{n+q}$. Furthermore, for $x_{0}=0$ in (18), we obtain, for any $T \in N$,

$$
\begin{aligned}
J^{T}(0, v):= & -\mathscr{E}\left[x^{T}(T+1) E^{T} \operatorname{PEx}(T+1)\right] \\
& +\sum_{t=0}^{T} \mathscr{E}\left\{\left[\begin{array}{l}
x(k) \\
v(k)
\end{array}\right]^{T} L(P)\left[\begin{array}{l}
x(k) \\
v(k)
\end{array}\right]\right\} \\
\leq & -\mathcal{E}^{2} \sum_{t=0}^{T} \mathscr{E}\|v(k)\|^{2} .
\end{aligned}
$$

Using (20), we derive

$$
\begin{aligned}
\sum_{k=0}^{\infty} \mathscr{E}\|z(k)\|^{2} & =J^{\infty}(0, v)+\gamma^{2} \sum_{k=0}^{\infty} \mathscr{E}\|v(k)\|^{2} \\
& \leq\left(\gamma^{2}-\varepsilon^{2}\right) \sum_{k=0}^{\infty} \mathscr{E}\|v(k)\|^{2} \\
& <\gamma^{2} \sum_{k=0}^{\infty} \mathscr{E}\|v(k)\|^{2}
\end{aligned}
$$

$$
\left[\begin{array}{cc}
-\bar{E}^{T} \overline{P E}+H e(\overline{F A})+\bar{A}_{0}^{T}\left[\bar{P}-\left(\bar{E}^{\perp}\right)^{T} \overline{Q E}^{\perp}\right] \bar{A}_{0} & -\bar{F}+\bar{A}^{T} \bar{G}^{T} \\
* & P-\left(\bar{E}^{\perp}\right)^{T} \overline{Q E}^{\perp}-\bar{G}-\bar{G}^{T}
\end{array}\right]<0,
$$

where

$$
\begin{aligned}
\bar{A} & =\left[\begin{array}{ll}
A & B_{1} \\
C & D
\end{array}\right], \\
\bar{E} & =\left[\begin{array}{cc}
E & 0 \\
0 & \gamma L_{p \times q}
\end{array}\right], \\
\bar{A}_{0} & =\left[\begin{array}{cc}
A_{0} & B_{0} \\
0 & 0
\end{array}\right], \\
\bar{P} & =\left[\begin{array}{cc}
P & 0 \\
0 & I_{p}
\end{array}\right], \\
\bar{Q} & =\left[\begin{array}{ll}
Q & 0 \\
0 & 0
\end{array}\right], \\
I_{q} & =L_{p \times q}^{T} L_{p \times q}, \quad(q \leq p) .
\end{aligned}
$$

Proof. (i) Let $X=P-\left(E^{\perp}\right)^{T} Q E^{\perp}$ in (22); then it is easy to test that the conditions of Theorem 11 are satisfied. So system
Theorem 12 (SBRL). System (1) with $u(k)=0$ is mean square admissible and $\|L\|<\gamma$ for given $\gamma>0$ if one of the following statements holds:

(i) There are matrices $P \in R^{n \times n}>0$ and $Q=Q^{T} \in$ $R^{(n-r) \times(n-r)}$ such that

$$
\left[\begin{array}{cc}
\Phi_{1} & \Phi_{2} \\
* & \Phi_{3}
\end{array}\right]<0
$$

where

$$
\begin{aligned}
\Phi_{1}= & A^{T}\left(P-E^{\perp^{T}} Q E^{\perp}\right) A+A_{0}^{T}\left(P-E^{\perp^{T}} Q E^{\perp}\right) A_{0} \\
& -E^{T} P E+C^{T} C, \\
\Phi_{2}= & A^{T}\left(P-E^{\perp T} Q E^{\perp}\right) B_{1}+A_{0}^{T}\left(P-E^{\perp^{T}} Q E^{\perp}\right) B_{0} \\
& +C^{T} D, \\
\Phi_{3}= & B_{1}^{T}\left(P-E^{\perp^{T}} Q E^{\perp}\right) B_{1}+B_{0}^{T}\left(P-E^{\perp^{T}} Q E^{\perp}\right) B_{0} \\
& -\gamma^{2} I+D^{T} D .
\end{aligned}
$$

(ii) There are matrices $P \in R^{n \times n}>0, Q=Q^{T} \in$ $R^{(n-r) \times(n-r)}, \bar{F}$ and $\bar{G}$ such that
(1) with $u(k)=0$ is mean square admissible and $\|L\|<\gamma$ for given $\gamma>0$.

(ii) It can be found that condition (22) can be rewritten as

$$
\begin{aligned}
& {\left[\begin{array}{ll}
A & B_{1} \\
C & D
\end{array}\right]^{T}\left[\begin{array}{cc}
X & 0 \\
0 & I_{p}
\end{array}\right]\left[\begin{array}{ll}
A & B_{1} \\
C & D
\end{array}\right]} \\
& +\left[\begin{array}{cc}
A_{0} & B_{0} \\
0 & 0
\end{array}\right]^{T}\left[\begin{array}{cc}
X & 0 \\
0 & I_{p}
\end{array}\right]\left[\begin{array}{cc}
A_{0} & B_{0} \\
0 & 0
\end{array}\right] \\
& -\left[\begin{array}{cc}
E & 0 \\
0 & \gamma L_{p \times q}
\end{array}\right]^{T}\left[\begin{array}{ll}
P & 0 \\
0 & I_{p}
\end{array}\right]\left[\begin{array}{cc}
E & 0 \\
0 & \gamma L_{p \times q}
\end{array}\right]<0 .
\end{aligned}
$$

Note that (26) is just the same as

$$
\begin{aligned}
\bar{A}^{T}[ & \left.\bar{P}-\left(\bar{E}^{\perp}\right)^{T} \overline{Q E}^{\perp}\right] \bar{A}+\bar{A}_{0}^{T}\left[\bar{P}-\left(\bar{E}^{\perp}\right)^{T} \overline{Q E}^{\perp}\right] \bar{A}_{0} \\
& -\bar{E}^{T} \overline{P E}<0 .
\end{aligned}
$$

Finally, we derive that (27) is equivalent to (24) by Theorem 7. 
Remark 13. When $E=I$, both Theorems 11 and 12 degenerate to SBRLs for state-space systems with state and disturbance dependent noise [5]. When $A_{0}=0$, system (1) reduces to a deterministic singular system. In this case, Theorems 12 and 14 are, respectively, consistent with the corresponding results of $[21,22]$ on deterministic singular systems. In addition, the additional condition $q \leq p$ in item (ii) needs to be satisfied, since only in this way can the expression $I_{q}=$ $L_{p \times q}^{T} L_{p \times q}$ hold. In contrast to item (i), item (ii) is somewhat complicated due to the introduction of the auxiliary matrices, but which provides an effective approach to investigate the $H_{\infty}$ controller design.

\section{4. $H_{\infty}$ State Feedback Controller Design}

In this section, we present a sufficient condition in the form of a strict LMI to design $H_{\infty}$ state feedback controller for system (1).
The discrete-time stochastic singular system with the state feedback control law $u(k)=K x(k)$ is given as

$$
\begin{aligned}
E x(k+1)= & (A+B K) x(k)+A_{0} x(k) w(k) \\
& +B_{1} v(k)+B_{0} v(k) w(k), \\
z(k)= & C x(k)+D v(k), \\
\operatorname{Ex}(0)= & x_{0} \in R^{n},
\end{aligned}
$$

$$
k \in N \text {, }
$$

where $K$ is a state feedback gain to be determined.

Theorem 14. The closed-loop system (28) is mean square admissible and $\|L\|<\gamma$ for given $\gamma>0$ if there exist matrices $\widehat{P} \in R^{(n+m) \times(n+m)}>0, \widehat{Q}=\widehat{Q}^{T} \in R^{(n+m-r) \times(n+m-r)}, \overline{\widehat{F}}$ and $\overline{\widehat{G}}$ such that the following LMI holds:

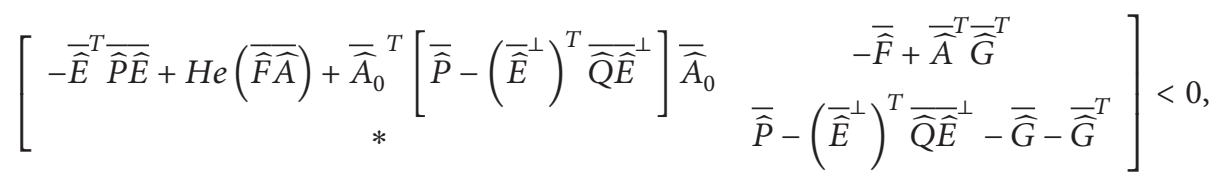

where

$$
\begin{aligned}
\overline{\widehat{E}} & =\left[\begin{array}{cc}
\widehat{E} & 0 \\
0 & \gamma L_{p \times q}
\end{array}\right], \\
\overline{\widehat{A}} & =\left[\begin{array}{cc}
\widehat{A} & \widehat{B}_{1} \\
\widehat{C} & D
\end{array}\right], \\
\widehat{\widehat{E}}^{\perp} & =\left[\begin{array}{cc}
\widehat{E}^{\perp} & 0 \\
0 & L_{p \times q}^{\perp}
\end{array}\right], \\
\overline{\widehat{A}} & =\left[\begin{array}{cc}
\widehat{A} & \widehat{B}_{0} \\
0 & 0
\end{array}\right], \\
\overline{\widehat{P}} & =\left[\begin{array}{cc}
\widehat{P} & 0 \\
0 & I_{p}
\end{array}\right], \\
\overline{\widehat{Q}} & =\left[\begin{array}{ll}
\widehat{Q} & 0 \\
0 & 0
\end{array}\right] .
\end{aligned}
$$

Proof. Let $\widehat{x}(k)=\left[\begin{array}{ll}x^{T}(k) u^{T}(k)\end{array}\right]^{T}$; then (28) can be written as an augmented system

$$
\begin{aligned}
\widehat{E} \widehat{x}(k+1)= & \widehat{A} \hat{x}(k)+\widehat{A}_{0} \widehat{x}(k) w(k)+\widehat{B}_{1} v(k) \\
& +\widehat{B}_{0} v(k) w(k), \\
z(k)= & \widehat{C} \hat{x}(k)+D v(k), \\
\widehat{E} \hat{x}(0)= & \widehat{x}_{0} \in R^{n},
\end{aligned}
$$

where

$$
\begin{aligned}
\widehat{E} & =\left[\begin{array}{ll}
E & 0 \\
0 & 0
\end{array}\right], \\
\widehat{A} & =\left[\begin{array}{ll}
A & B \\
K & -I_{m}
\end{array}\right], \\
\widehat{A}_{0} & =\left[\begin{array}{ll}
A_{0} & 0 \\
0 & 0
\end{array}\right], \\
\widehat{B}_{1} & =\left[\begin{array}{l}
B_{1} \\
0
\end{array}\right], \\
\widehat{B} & =\left[\begin{array}{c}
B_{0} \\
0
\end{array}\right], \\
\widehat{C} & =\left[\begin{array}{ll}
C & 0
\end{array}\right] .
\end{aligned}
$$

It is noted that if there exist matrices $U, V$ such that (6) holds, then we have

$$
\begin{aligned}
\widehat{U} \widehat{E} \widehat{V} & =\left[\begin{array}{lll}
I_{r} & 0 & 0 \\
0 & 0 & 0 \\
0 & 0 & 0
\end{array}\right], \\
\widehat{U} \widehat{A}_{0} \widehat{V} & =\left[\begin{array}{ccc}
\widehat{A}_{01} & \widehat{A}_{02} & 0 \\
0 & 0 & 0 \\
0 & 0 & 0
\end{array}\right],
\end{aligned}
$$




$$
\widehat{U} \widehat{B}_{0}=\left[\begin{array}{c}
\widehat{B}_{01} \\
0 \\
0
\end{array}\right] \text {, }
$$

with

$$
\begin{aligned}
& \widehat{U}=\left[\begin{array}{ll}
U & 0 \\
0 & U
\end{array}\right], \\
& \widehat{V}=\left[\begin{array}{ll}
V & 0 \\
0 & V
\end{array}\right] .
\end{aligned}
$$

That is to say, the assumption condition for system (31) still holds. Therefore, from Theorem 12-(ii), the closed-loop system (28) is mean square admissible and $\|L\|<\gamma$ for given $\gamma>0$ if the condition (29) holds.

Remark 15. To solve the LMI condition (29), particular structures of matrices $\overline{\widehat{F}}$ and $\overline{\widehat{G}}$ were given as that in [22]:

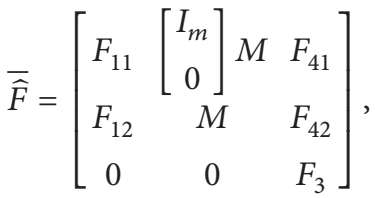

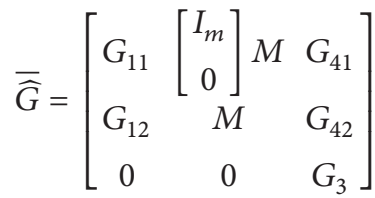

with $F_{11} \in R^{n \times n}, F_{12} \in R^{m \times n}, F_{41} \in R^{n \times p}, F_{42} \in R^{m \times p}, F_{3} \in$ $R^{q \times p}, G_{11} \in R^{n \times n}, G_{12} \in R^{m \times n}, G_{41} \in R^{n \times p}, G_{42} \in R^{m \times p}, G_{3} \in$ $R^{q \times p}$, and a nonsingular matrix $M \in R^{m \times m}$. Set $N=M K$; then the state feedback gain matrix is given as $K=M^{-1} N$.

Remark 16. It should be pointed out that what we have obtained such as Theorems 12 and 14 are corresponding extensions from deterministic singular systems to stochastic singular systems. Although a strict LMI-based bounded real lemma was presented in [21] for discrete-time and deterministic singular systems, it is difficult to be generalized to $H_{\infty}$ controller synthesis. While by introducing the auxiliary matrices, the state feedback $H_{\infty}$ control for stochastic singular systems is successfully solved in Theorem 14 . Moreover, in contrast with the state feedback control, the output feedback control has much broader and practical applications such as [23]. Therefore, it is very meaningful to extend the results of this paper to the output feedback control, which merits further research.

\section{Numerical Examples}

In this section, we give a numerical example to show the effectiveness of the proposed results. The discrete-time stochastic singular system with the state feedback control $u(k)=K x(k)$ in the form of (28) takes the following data:

$$
\begin{aligned}
E & =\left[\begin{array}{ll}
1 & 2 \\
2 & 4
\end{array}\right], \\
A & =\left[\begin{array}{ll}
2.5 & 1.0 \\
1.7 & 0.8
\end{array}\right], \\
A_{0} & =\left[\begin{array}{ll}
0.1 & 0.2 \\
0.2 & 0.4
\end{array}\right], \\
B & =\left[\begin{array}{l}
0.1 \\
0
\end{array}\right], \\
B_{1} & =\left[\begin{array}{l}
0.1 \\
0.3
\end{array}\right], \\
B_{0} & =\left[\begin{array}{l}
0.3 \\
0.6
\end{array}\right], \\
C & =\left[\begin{array}{ll}
0.6 & 0.7
\end{array}\right], \\
D & =0.01, \\
E^{\perp} & =\left[\begin{array}{ll}
0.2 & -0.1
\end{array}\right], \\
\gamma & =0.56 .
\end{aligned}
$$

From Theorem 14, the solutions of LMI (29) are given as follows:

$$
\begin{aligned}
\widehat{P} & =\left[\begin{array}{ccc}
0.6039 & -0.2789 & 0.1258 \\
-0.2789 & 0.2617 & 0.0260 \\
0.1258 & 0.0260 & 0.3139
\end{array}\right], \\
\widehat{Q} & =85.7046, \\
\overline{\widehat{F}} & =\left[\begin{array}{cccc}
-0.6040 & 0.5637 & 0.4307 & 0.5739 \\
-0.0011 & 0.2152 & 0 & 0.7034 \\
-0.0034 & 0.0512 & 0.4307 & -0.0394 \\
0 & 0 & 0 & -0.0791
\end{array}\right], \\
\overline{\widehat{G}} & =\left[\begin{array}{cccc}
-1.0056 & 1.0094 & 0.4307 & 0.1096 \\
0.2592 & 0.0001 & 0 & -0.0442 \\
-0.0803 & 0.2297 & 0.4307 & 0.0118 \\
0 & 0 & 0 & 0.8987
\end{array}\right], \\
M & =0.4307, \\
N & =\left[\begin{array}{llll}
0.1071 & -0.0208
\end{array}\right] .
\end{aligned}
$$

Then the controller gain is given as $K=M^{-1} N=$ [0.2486 -0.0483].

In what follows, it can be seen from Figures 1 and 2 that the curves are convergent which demonstrates the closed system (28) without external disturbance is stabilizable in 


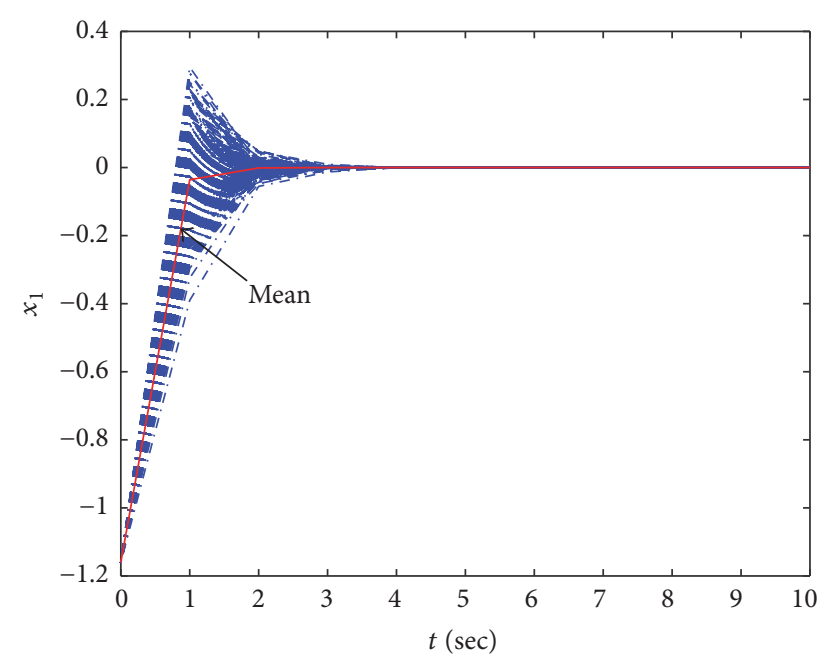

FIgURE 1: Trajectory of $x_{1}(k)$ : the closed-loop system (28) without external disturbance.

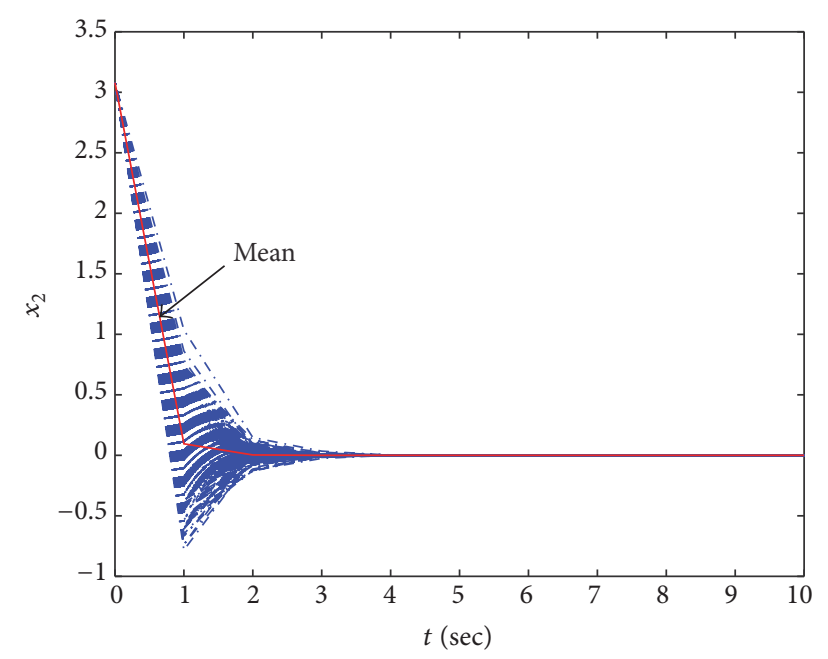

FIGURE 2: Trajectory of $x_{2}(k)$ : the closed-loop system (28) without external disturbance.

the mean square via the controller $u(k)=K x(k)$. Figure 3 shows that the maximal possible energy gain from the external disturbance to the output for system (28) is less than the prescribed $H_{\infty}$ performance level $\gamma=0.56$; that is, the requirement of the $H_{\infty}$ performance of system (28) is satisfied.

\section{Conclusion}

In this paper, we have discussed the stochastic $H_{\infty}$ state feedback control problem for a class of discrete-time singular systems with state and disturbance dependent noise. New conditions for discrete-time stochastic singular systems to be mean square admissible have been derived in strict LMIs. It is proved that the state $x(k)$ is uncorrelated with the stochastic variable sequence $\{w(k)\}_{k \geq 0}$. Based on this result, two strict LMI-based SBRLs are then presented. By introducing the auxiliary matrix technique, the state feedback $H_{\infty}$ control problem has been addressed. What we have obtained can be used further to study other control problems of discretetime singular stochastic systems, such as the output feedback control and $\mathrm{H}_{2} / \mathrm{H}_{\infty}$ control.

\section{Appendix}

Proof of Lemma 6. The proof of item (i) sees Theorem 5 of [37]. Next, we set about to verify item (ii).

Based on Assumption 5, there exist nonsingular matrices $U, V$ such that

$$
\begin{aligned}
U E V & =\left[\begin{array}{ll}
I_{r} & 0 \\
0 & 0
\end{array}\right], \\
U A V & =\left[\begin{array}{ll}
\widehat{A}_{11} & \widehat{A}_{12} \\
\widehat{A}_{21} & \widehat{A}_{22}
\end{array}\right], \\
U A_{0} V & =\left[\begin{array}{cc}
\widehat{A}_{01} & \widehat{A}_{02} \\
0 & 0
\end{array}\right], \\
U B_{1} & =\left[\begin{array}{c}
\widehat{B}_{11} \\
\widehat{B}_{21}
\end{array}\right], \\
U B_{0} & =\left[\begin{array}{c}
\widehat{B}_{01} \\
0
\end{array}\right] .
\end{aligned}
$$

Let

$$
U^{-T} P U^{-1}=\left[\begin{array}{ll}
\widehat{P}_{11} & \widehat{P}_{12} \\
\widehat{P}_{12}^{T} & \widehat{P}_{22}
\end{array}\right],
$$

and substituting (A.1) and (A.3) into (7) and (8), respectively, we derive

$$
\begin{aligned}
& E^{T} P E=\left(V^{-T}\left[\begin{array}{cc}
I_{r} & 0 \\
0 & 0
\end{array}\right] U^{-T}\right)\left(U^{T}\left[\begin{array}{cc}
\widehat{P}_{11} & \widehat{P}_{12} \\
\widehat{P}_{12}^{T} & \widehat{P}_{22}
\end{array}\right] U\right) \\
& \cdot\left(U^{-1}\left[\begin{array}{cc}
I_{r} & 0 \\
0 & 0
\end{array}\right] V^{-1}\right)=V^{-T}\left[\begin{array}{cc}
\widehat{P}_{11} & 0 \\
0 & 0
\end{array}\right] V^{-1} \geq 0 \\
& A^{T} P A+A_{0}^{T} P A_{0}-E^{T} P E=V^{-T}\left[\begin{array}{cc}
M_{1} & M_{2} \\
M_{2}^{T} & M_{3}
\end{array}\right] V^{-1} \\
& <0
\end{aligned}
$$

where

$$
\begin{aligned}
M_{1}= & \widehat{A}_{11}^{T} \widehat{P}_{11} \widehat{A}_{11}+\widehat{A}_{01}^{T} \widehat{P}_{11} \widehat{A}_{01}+\widehat{A}_{21}^{T} \widehat{P}_{12}^{T} \widehat{A}_{11} \\
& +\widehat{A}_{11}^{T} \widehat{P}_{12} \widehat{A}_{21}+\widehat{A}_{21}^{T} \widehat{P}_{12} \widehat{A}_{21}-\widehat{P}_{11}, \\
M_{2}= & \widehat{A}_{11}^{T} \widehat{P}_{11} \widehat{A}_{12}+\widehat{A}_{01}^{T} \widehat{P}_{11} \widehat{A}_{02}+\widehat{A}_{21}^{T} \widehat{P}_{12}^{T} \widehat{A}_{12} \\
& +\widehat{A}_{11}^{T} \widehat{P}_{12} \widehat{A}_{22}+\widehat{A}_{21}^{T} \widehat{P}_{22} \widehat{A}_{22}, \\
M_{3}= & \widehat{A}_{12}^{T} \widehat{P}_{11} \widehat{A}_{12}+\widehat{A}_{02}^{T} \widehat{P}_{11} \widehat{A}_{02}+\widehat{A}_{22}^{T} \widehat{P}_{12}^{T} \widehat{A}_{12} \\
& +\widehat{A}_{12}^{T} \widehat{P}_{12} \widehat{A}_{22}+\widehat{A}_{22}^{T} \widehat{P}_{22} \widehat{A}_{22} .
\end{aligned}
$$



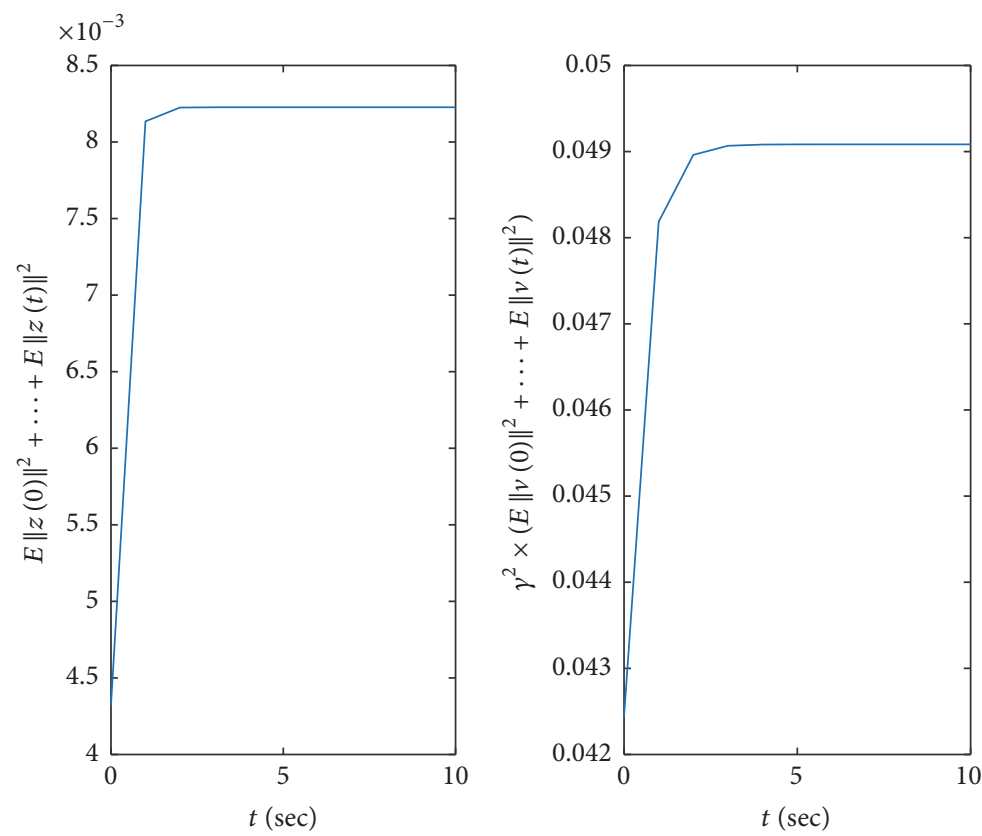

FIGURE 3: $H_{\infty}$ performance analysis: the closed-loop system (28) without external disturbance.

Equation (A.5) implies $M_{3}<0$. Furthermore, since $\widehat{P}_{11} \geq 0$, we have

$$
\widehat{A}_{12}^{T} \widehat{P}_{11} \widehat{A}_{12}+\widehat{A}_{02}^{T} \widehat{P}_{11} \widehat{A}_{02} \geq 0 .
$$

Therefore, in $M_{3}$, it is easy to see that

$$
\widehat{A}_{22}^{T} \widehat{P}_{12}^{T} \widehat{A}_{12}+\widehat{A}_{12}^{T} \widehat{P}_{12} \widehat{A}_{22}+\widehat{A}_{22}^{T} \widehat{P}_{22} \widehat{A}_{22}<0 .
$$

From (A.8), it follows that $\widehat{A}_{22}$ is invertible. Letting

$$
\widehat{V}=V\left[\begin{array}{cc}
I_{r} & 0 \\
-\widehat{A}_{22}^{-1} \widehat{A}_{21} & \widehat{A}_{22}^{-1}
\end{array}\right],
$$

we derive

$$
\begin{aligned}
U E \widehat{V} & =\left[\begin{array}{ll}
I_{r} & 0 \\
0 & 0
\end{array}\right], \\
U A \widehat{V} & =\left[\begin{array}{cc}
\widehat{A}_{11}-\widehat{A}_{12} \widehat{A}_{22}^{-1} \widehat{A}_{21} & \widehat{A}_{12} \widehat{A}_{22}^{-1} \\
0 & I_{n-r}
\end{array}\right], \\
U A_{0} \widehat{V} & =\left[\begin{array}{cc}
\widehat{A}_{01}-\widehat{A}_{02} \widehat{A}_{22}^{-1} \widehat{A}_{21} & \widehat{A}_{02} \widehat{A}_{22}^{-1} \\
0 & 0
\end{array}\right] .
\end{aligned}
$$

Let

$$
x(k)=\widehat{V}\left[\begin{array}{ll}
\xi_{1}(k)^{T} & \xi_{2}(k)^{T}
\end{array}\right]^{T},
$$

where $\xi_{1}(k) \in R^{r}, \xi_{2}(k) \in R^{n-r}$. By (A.2), (A.10), and (A.11), system (1) with $u(k)=0$ is equivalent to

$$
\begin{aligned}
\xi_{1}(k+1)= & \widetilde{A}_{1} \xi_{1}(k)+\widetilde{A}_{01} \xi_{1}(k) w(k) \\
& +\left(\widehat{B}_{11}-\widehat{A}_{12} \widehat{A}_{22}^{-1} \widehat{B}_{21}\right) v(k) \\
& +\left(\widehat{B}_{01}-\widehat{A}_{02} A_{22}^{-1} \widehat{B}_{21}\right) v(k) w(k), \\
\xi_{2}(k)= & -\widehat{B}_{21} v(k),
\end{aligned}
$$

with

$$
\begin{gathered}
\widetilde{A}_{1}=\widehat{A}_{11}-\widehat{A}_{12} \widehat{A}_{22}^{-1} \widehat{A}_{21}, \\
\widetilde{A}_{01}=\widehat{A}_{01}-\widehat{A}_{02} \widehat{A}_{22}^{-1} \widehat{A}_{21} .
\end{gathered}
$$

Since $\{w(k)\}_{k \geq 0}$ is a sequence of real independent random variables, considering (A.12), we have that $\xi_{1}(k)$ that is $F_{k-1}$ measurable is uncorrelated with $w(k)$, so does $x(k)$.

\section{Disclosure}

This paper was not presented at any IFAC meeting.

\section{Conflicts of Interest}

The authors declare that they have no conflicts of interest.

\section{Acknowledgments}

This research was supported by NSF of China under Grant no. 61573227, State Key Laboratory of Alternate Electrical Power System with Renewable Energy Sources (Grant no. LAPS16011), and the SDUST Research Fund no. 2015 TDJH105. 


\section{References}

[1] X. Mao, Stochastic Differential Equations and Their Applications, Horwood Publishing Limited, Chichester, UK, 1997.

[2] B. Øksendal, Stochastic Differential Equations: An Introduction with Applications, Springer, New York, NY, USA, 1998.

[3] V. Dragan, T. Morozan, and A. M. Stoica, Mathematical Methods in Robust Control of Linear Stochastic Systems, Springer, New York, NY, USA, 2006.

[4] V. Dragan, T. Morozan, and A.-M. Stoica, Mathematical Methods in Robust Control of Discrete-Time Linear Stochastic Systems, Springer, New York, NY, USA, 2010.

[5] A. El Bouhtouri, D. Hinrichsen, and A. J. Pritchard, " $H_{\infty}$ type control for discrete-time stochastic systems," International Journal of Robust and Nonlinear Control, vol. 9, no. 13, pp. 923948, 1999.

[6] D. Hinrichsen and A. J. Pritchard, "Stochastic $H_{\infty}$ ", SIAM Journal on Control and Optimization, vol. 36, no. 5, pp. 15041538, 1998.

[7] B. Chen and W. Zhang, "Stochastic $\mathrm{H}_{2} / \mathrm{H}_{\infty}$ control with statedependent noise," IEEE Transactions on Automatic Control, vol. 49, no. 1, pp. 45-57, 2004.

[8] W. Zhang, Y. Huang, and H. Zhang, "Stochastic $\mathrm{H}_{2} / \mathrm{H}_{\infty}$ control for discrete-time systems with state and disturbance dependent noise," Automatica, vol. 43, no. 3, pp. 513-521, 2007.

[9] W. Zhang, Y. Huang, and L. Xie, "Infinite horizon stochastic $\mathrm{H}_{2} / \mathrm{H}_{\infty}$ control for discrete-time systems with state and disturbance dependent noise," Automatica, vol. 44, no. 9, pp. 23062316, 2008.

[10] W. Zhang, X. Lin, and B. Chen, "LaSalle-type theorem and its applications to infinite horizon optimal control of discrete-time nonlinear stochastic systems," IEEE Transactions on Automatic Control, vol. 62, no. 1, pp. 250-261, 2017.

[11] D. G. Luenberger and A. Arbel, "Singular dynamic leontief systems," Econometrica, vol. 45, no. 4, pp. 991-995, 1977.

[12] F. L. Lewis, "A survey of linear singular systems," Circuits, Systems and Signal Processing, vol. 5, no. 1, pp. 3-36, 1986.

[13] L. Dai, Singular Control Systems, vol. 118 of Lecture Notes in Control and Information Sciences, Springer, New York, NY, USA, 1989.

[14] L. Zhang, J. Lam, and Q. Zhang, "Lyapunov and Riccati equations of discrete-time descriptor systems," IEEE Transactions on Automatic Control, vol. 44, no. 11, pp. 2134-2139, 1999.

[15] S. Xu and J. Lam, Robust Control and Filtering of Singular Systems, Springer, Berlin, Germany, 2006.

[16] Y. Xia, J. Zhang, and E.-K. Boukas, "Control for discrete singular hybrid systems," Automatica, vol. 44, no. 10, pp. 2635-2641, 2008.

[17] Y. Xia, E.-K. Boukas, P. Shi, and J. Zhang, "Stability and stabilization of continuous-time singular hybrid systems," Automatica, vol. 45, no. 6, pp. 1504-1509, 2009.

[18] I. Masubuchi, Y. Kamitane, A. Ohara, and N. Suda, " $H_{\infty}$ control for descriptor systems: a matrix inequalities approach," Automatica, vol. 33, no. 4, pp. 669-673, 1997.

[19] S. Xu and C. Yang, " $H_{\infty}$ state feedback control for discrete-time singular systems," IEEE Xplore: IEEE Transactions on Automatic Control, vol. 45, no. 7, pp. 1405-1409, 2000.

[20] X. Ji, H. Su, and J. Chu, "Robust state feedback $\mathrm{H}_{\infty}$ control for uncertain linear discrete singular systems," IET Control Theory and Applications, vol. 1, no. 1, pp. 195-200, 2007.

[21] G. Zhang, Y. Xia, and P. Shi, "New bounded real lemma for discrete-time singular systems," Automatica, vol. 44, no. 3, pp. 886-890, 2008.
[22] M. Chadli and M. Darouach, "Novel bounded real lemma for discrete-time descriptor systems: application to $\mathrm{H}_{\infty}$ control design," Automatica, vol. 48, no. 2, pp. 449-453, 2012.

[23] M. Chadli and M. Darouach, "Further enhancement on robust control design for discrete-time singular systems," IEEE Transactions on Automatic Control, vol. 59, no. 2, pp. 494-499, 2014.

[24] M. Chadli, H. R. Karimi, and P. Shi, "On stability and stabilization of singular uncertain Takagi-Sugeno fuzzy systems," Journal of the Franklin Institute, vol. 351, no. 3, pp. 1453-1463, 2014.

[25] M. Chadli and M. Darouach, "Robust admissibility of uncertain switched singular systems," International Journal of Control, vol. 84, no. 10, pp. 1587-1600, 2011.

[26] L. Zhang, B. Huang, and J. Lam, "LMI synthesis of $\mathrm{H}_{2}$ and mixed $\mathrm{H}_{2} / \mathrm{H}_{\mathrm{\infty}}$ controllers for singular systems," IEEE Transactions on Circuits and Systems II, vol. 50, no. 9, pp. 615-626, 2003.

[27] L. Wu, P. Shi, and H. Gao, "State estimation and slidingmode control of Markovian jump singular systems," IEEE Transactions on Automatic Control, vol. 55, no. 5, pp. 1213-1219, 2010.

[28] L. Wu, X. Su, and P. Shi, "Sliding mode control with bounded $\mathscr{L}_{2}$ gain performance of Markovian jump singular time-delay systems," Automatica, vol. 48, no. 8, pp. 1929-1933, 2012.

[29] E. K. Boukas, "Stabilization of stochastic singular nonlinear hybrid systems," Nonlinear Analysis: Theory, Methods \& Applications, vol. 64, no. 2, pp. 217-228, 2006.

[30] L. Wu and D. W. C. Ho, "Sliding mode control of singular stochastic hybrid systems," Automatica, vol. 46, no. 4, pp. 779783, 2010.

[31] D. W. C. Ho, X. Shi, Z. Wang, and Z. Gao, "Filtering for a class of stochastic descriptor systems," in Proceedings of the 4th International Conference on Dynamics of Continuous, Discrete and Impulsive Systems, no. 2, pp. 848-853, Ontario, Canada, 2005.

[32] J. Xia, Robust control and filter for continuous stochastic timedelay systems [Ph.D. thesis], Nanjing University of Science and Technology, Nanjing, China, 2007.

[33] L. Huang and X. Mao, "Stability of singular stochastic systems with Markovian switching," IEEE Transactions on Automatic Control, vol. 56, no. 2, pp. 424-429, 2011.

[34] Z. Gao and X. Shi, "Observer-based controller design for stochastic descriptor systems with Brownian motions," Automatica, vol. 49, no. 7, pp. 2229-2235, 2013.

[35] S. Xing, Q. Zhang, S. Yuan, J. Wang, and S. Qian, "Robust finitetime $\mathrm{H}_{\infty}$ control for uncertain stochastic singular systems," in Proceedings of the 33th Chinese Control Conference, Nanjing, China, July 2014.

[36] W. Zhang, Y. Zhao, and L. Sheng, "Some remarks on stability of stochastic singular systems with state-dependent noise," Automatica, vol. 51, pp. 273-277, 2015.

[37] Y. Zhao and W. Zhang, "New results on stability of singular stochastic Markov jump systems with state-dependent noise," International Journal of Robust and Nonlinear Control, vol. 26, no. 10, pp. 2169-2186, 2016.

[38] Y. Zhao and W. Zhang, "Observer-based controller design for singular stochastic Markov jump systems with state dependent noise," Journal of Systems Science \& Complexity, vol. 29, no. 4, pp. 946-958, 2016.

[39] Y. Zhao and W. Zhang, "Stochastic state feedback $H_{\infty}$ control for singular systems with multiplicative noise," in Proceedings of the 35th Chinese Control Conference, Chengdu, China, July 2016. 


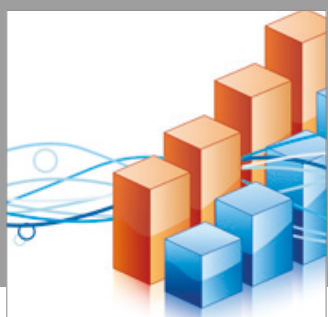

Advances in

Operations Research

vatersals

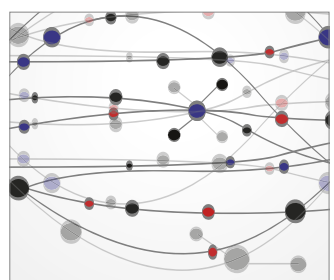

\section{The Scientific} World Journal
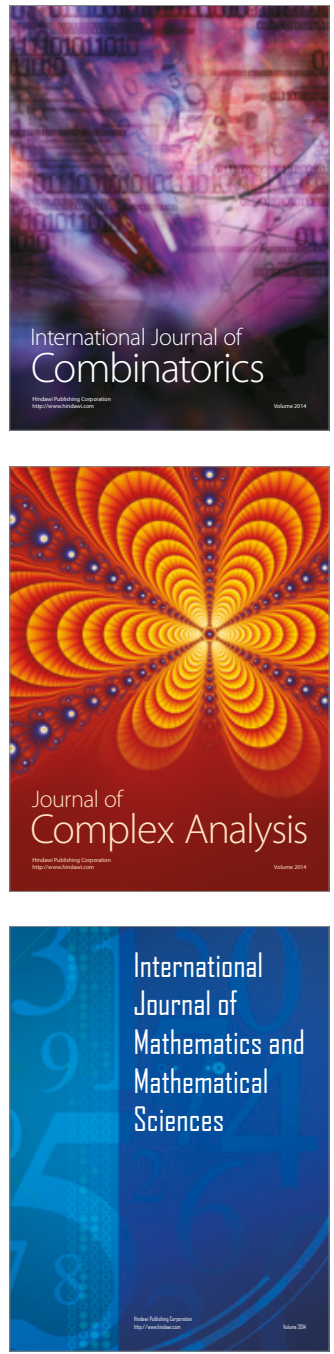
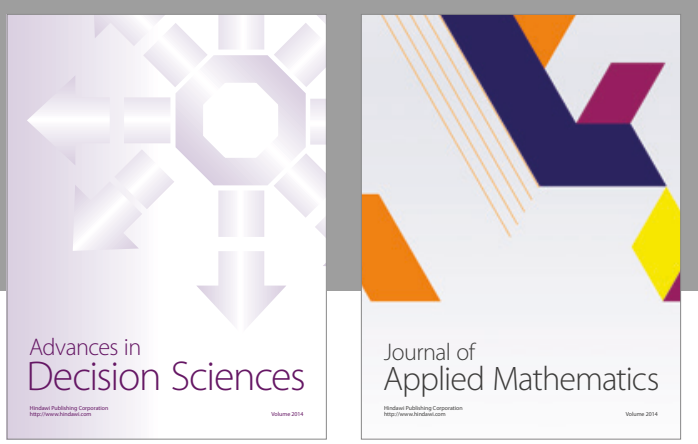

Algebra

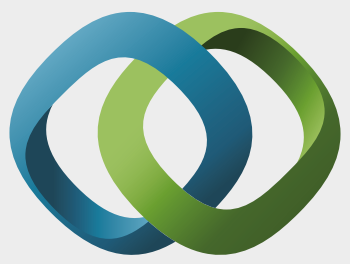

\section{Hindawi}

Submit your manuscripts at

https://www.hindawi.com
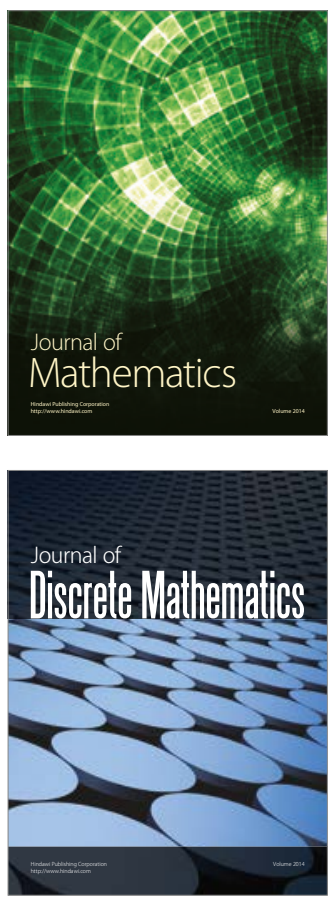

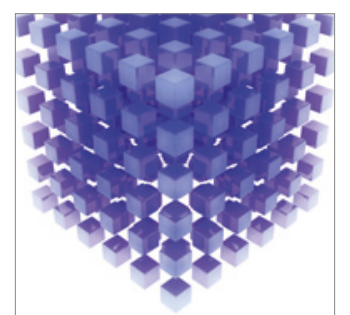

Mathematical Problems in Engineering
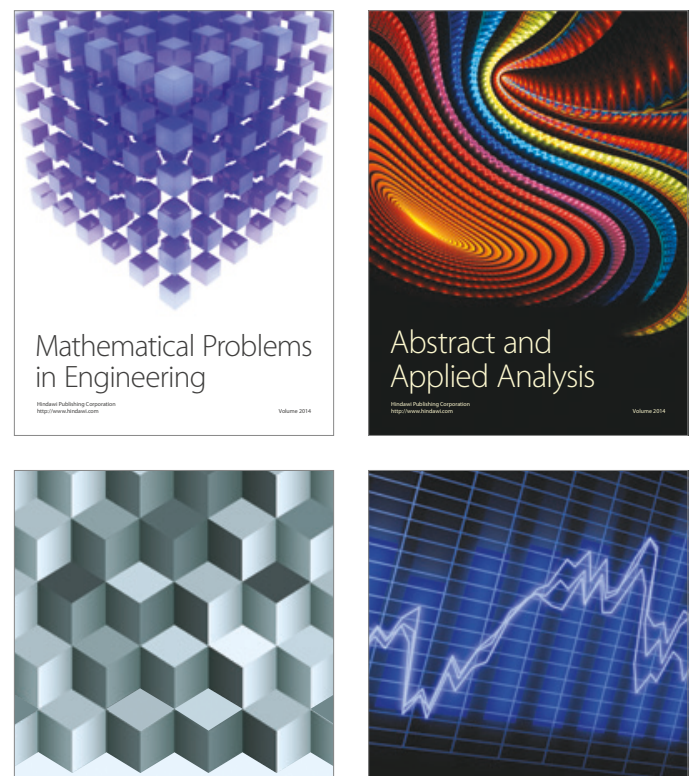

Journal of

Function Spaces

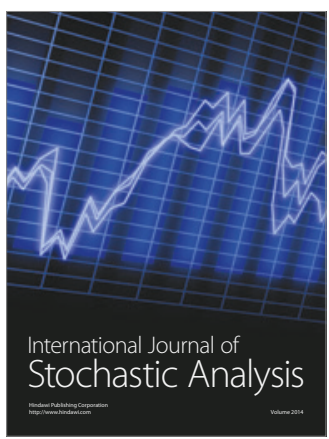

Probability and Statistics
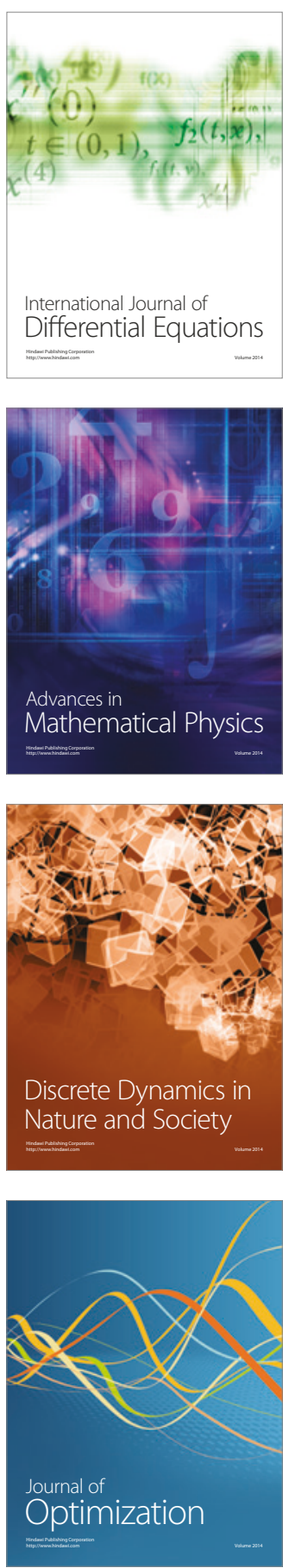